\title{
Food choices and life expectancy: is it just beans?
}

Vegard Lysne ${ }^{1,2,3}$, Thomas Olsen ${ }^{6}$, Theogene Habumugisha ${ }^{3,5}$, Ingunn M.S. Engebretsen ${ }^{3,5}$, Jutta Dierkes ${ }^{1,2,4}$

${ }^{1}$ University of Bergen, MOHN Nutrition research laboratory, and ${ }^{2}$ Centre for Nutrition, Bergen Norway

${ }^{3}$ Department of Heart Disease, and ${ }^{4}$ Department of Laboratory Medicine and Pathology, Haukeland University Hospital, Bergen, Norway

${ }^{5}$ Centre for International Health, Department of Global Public Health and Primary Care, University of Bergen, Norway

${ }^{6}$ University of Oslo, Institute of Basic Medical Science, Oslo, Norway

In response to: Fadnes $\mathrm{LT}, \varnothing \mathrm{kland} \mathrm{J}-\mathrm{M}$, Haaland $\varnothing \mathrm{A}$, Johansson KA. Estimating impact of food choices on life expectancy: A modeling study. PLoS Med 2022; 19: e1003889.

https://doi.org/10.1371/journal.pmed.1003889 
A modeling study by Fadnes et al just published in PLOS Medicine has amassed considerable news and social media attention for its claim that an optimized diet could lead to as much as 13 additional years of life expectancy. ${ }^{1}$ At the time of writing, the study has been viewed more than 120,000 times and generated 247 generally favorable news stories including major news outlets such as CNN and The Telegraph. Although the research effort is ambitious and admirable, the messages that have reached the general public are grossly oversimplified from a nutritional point of view and we have substantial reservations about the methodological approach, interpretations, and conclusions from this study.

The study additively combines effect estimates from published meta-analyses on diet-disease associations for individual foods and food groups to estimate total life-years gained by sustained dietary changes. Although we agree that transitioning to a healthy diet will result in healthy life years gains, we consider the estimates reported in the paper and thereafter in news headlines as implausible for several reasons. Firstly, any changes in energy intake are neglected. This is one of the most important components of dietary exposure and a crucial determinant of health both in under- and overnutrition. Secondly, the effects of changing the intake of individual foods are treated separately and independently, practically ignoring food substitution effects which is a key concept in nutrition research. ${ }^{2}$ Whenever the intake of one food changes, this is inevitably followed by changes in the intake of other foods and in most cases total energy intake. Thus, health effects must always be interpreted in light of which food is replaced. Thirdly, the individual effect estimates are highly overlapping and influenced by both socioeconomic status (SES) and health consciousness, which are major determinants of longevity. When additively combined, this inevitably causes the model to overestimate the total effect.

We also question the validity of the dietary intake estimates used to calculate the expected effect of specific dietary changes. Food frequency questionnaires (FFQ) are the dietary tool most used for the underlying data. FFQs are mainly used to rank individuals according to dietary exposure, and thus well suited for relative comparisons across different intake levels. However, estimates of absolute intakes should be interpreted with caution due to the presence of systematic measurement error. The model developed by Fadnes et al gives the impression of being able to predict the effect of very specific food intake levels, and we are concerned that this could be interpreted literally by the end-user.

Further, we cannot reasonably assume that the health effects of any food are homogeneous across all population groups, i.e. there is no such thing as 'one size fits all' in nutrition. Among other things, the model does not adequately consider acceptability and the situation in lowand middle-income countries (LMICS) or the growing concern of undernutrition among vulnerable subgroups of the population. A fitting example for the issue of LMICs is the PURE study, where diet-disease associations oppose what has been typically observed in Western populations. ${ }^{3}$ However, baseline nutrition status and food intake in LMICs differ from what is observed in Western affluent populations, and different foods are associated with higher SES. 
In contrast to the EAT-Lancet commission's healthy diet from sustainable food systems ${ }^{4}$, the optimal diet described in this paper eliminates red meat and drastically increases legumes and fish above and beyond what is practically feasible or sustainable. Indeed, eliminating intakes of entire food groups could do more harm than good for specific subgroups. For instance, lowering red meat intake would most likely be beneficial for the majority. ${ }^{5,6}$ At the same time, as a considerable source of protein, energy, and many micronutrients, the effect is likely not the same in populations at risk of undernutrition, such as the elderly, as we previously suggested ${ }^{7}$. As emphasized by EAT-Lancet, moderate meat consumption can provide adequate nutrients ${ }^{4}$, and it can be obtained from sustainably produced livestock. ${ }^{8}$

Healthy skepticism has been advised when interpreting observational research on preventive interventions, especially when results appear surprisingly large. ${ }^{9}$ Unfortunately, we think that the results from Fadnes et al join the ranks of implausible effects. The authors clearly state, and we agree, that the model is not meant for individualized forecasting. Nonetheless, they go on to conclude that clinicians, policymakers, and laypeople could use the calculator to understand the effect of dietary choices. Although we consider the estimated gains in life expectancy as exaggerated, we agree that dietary changes in the general direction described in the paper would benefit the majority in the Western world and would imply reduced meat intake and increased vegetable, legumes, and fish intakes. This includes huge changes for the food systems and questions the sustainability of fish stocks. ${ }^{4}$ For large parts of the world's population who have fewer choices improved food security and diversity are a necessity, in combination with a sustainable shift in the diet in the Western world, in order to achieve the sustainable development goal of Zero Hunger. 


\section{References:}

1 Fadnes LT, $\varnothing$ kland J-M, Haaland $\varnothing \mathrm{A}$, Johansson KA. Estimating impact of food choices on life expectancy: A modeling study. PLoS Med 2022; 19: e1003889.

2 Ibsen DB, Laursen ASD, Würtz AML, et al. Food substitution models for nutritional epidemiology. Am J Clin Nutr 2020; published online Dec 9. DOI:10.1093/ajcn/nqaa315.

3 Dehghan M, Mente A, Zhang X, et al. Associations of fats and carbohydrate intake with cardiovascular disease and mortality in 18 countries from five continents (PURE): a prospective cohort study. Lancet (London, England) 2017; 390: 2050-62.

4 Willett W, Rockström J, Loken B, et al. Food in the Anthropocene: the EAT-Lancet Commission on healthy diets from sustainable food systems. Lancet (London, England) 2019; 393: 447-92.

5 Farvid MS, Sidahmed E, Spence ND, Mante Angua K, Rosner BA, Barnett JB. Consumption of red meat and processed meat and cancer incidence: a systematic review and metaanalysis of prospective studies. Eur J Epidemiol 2021; 36: 937-51.

6 Papier K, Fensom GK, Knuppel A, et al. Meat consumption and risk of 25 common conditions: outcome-wide analyses in 475,000 men and women in the UK Biobank study. BMC Med 2021; 19. DOI:10.1186/S12916-021-01922-9.

7 Matre ÅO, Van Parys A, Olsen T, et al. The Association of Meat Intake With All-Cause Mortality and Acute Myocardial Infarction Is Age-Dependent in Patients With Stable Angina Pectoris. Front Nutr 2021; 8. DOI:10.3389/fnut.2021.642612.

8 Röös E, Patel M, Spångberg J, Carlsson G, Rydhmer L. Limiting livestock production to pasture and by-products in a search for sustainable diets. Food Policy 2016; 58: 1-13.

9 Shrank WH, Patrick AR, Brookhart MA. Healthy User and Related Biases in Observational Studies of Preventive Interventions: A Primer for Physicians. J Gen Intern Med 2011; 26: 546. 